Gut and Liver, Vol. 10, No. 6, November 2016, pp. 948-954

\title{
The Combination of Periostin Overexpression and Microvascular Invasion Is Related to a Poor Prognosis for Hepatocellular Carcinoma
}

\author{
Se Young Jang ${ }^{1}$, Soo Young Park ${ }^{1}$, Hye Won Lee ${ }^{2}$, Yeon-Kyung Choi ${ }^{1}$, Keun-Gyu Park ${ }^{1}$, Ghil Suk Yoon ${ }^{2}$, Won Young Tak ${ }^{1}$, \\ Young Oh Kweon ${ }^{1}$, Keun Hur ${ }^{3,4}$, and Won Kee Lee \\ Departments of ${ }^{1}$ Internal Medicine and ${ }^{2}$ Pathology, Kyungpook National University Hospital, ${ }^{3}$ Department of Biochemistry and Cell Biology, \\ Cell and Matrix Research Institute, Kyungpook National University School of Medicine, ${ }^{4}$ BK21 Plus KNU Biomedical Convergence Program, \\ Department of Biomedical Science, Kyungpook National University, and ${ }^{5}$ Biostatistics Center, Kyungpook National University School of \\ Medicine, Daegu, Korea
}

\section{See editorial on page 871.}

Background/Aims: Periostin is an extracellular matrix protein and is known to be related to the metastatic potential and prognosis of cancer. However, few studies have investigated the expression level of periostin and its association with prognoses in hepatocellular carcinoma. Therefore, we analyzed periostin overexpression in hepatocellular carcinoma and its implication for prognoses. Methods: We evaluated 149 patients who underwent surgical resection between 2006 and 2010. Tissue microarrays were constructed from hepatocellular carcinoma tissue and adjacent nontumor tissue, and immunohistochemistry was performed. Results: A high periostin level was observed more frequently in cases of multiple tumors (odds ratio [OR], 2.826; 95\% confidence interval [Cl], 1.224 to $6.527 ; p=0.013$ ), positive microvascular invasion (OR, 2.974; 95\% Cl, 1.431 to $6.181 ; \mathrm{p}=0.003$ ), and advanced stage disease (OR, 3.032; 95\% Cl, 1.424 to 6.452; $p=0.003)$. Patients with high periostin expression had significantly $(p=0.002)$ lower overall survival rates than those with low periostin expression $(90.3 \%, 66.1 \%$, and $56.2 \%$ vs $97.7 \%, 85.1 \%$, and $77.5 \%$ at 1,3 , and 5 years). Conclusions: We found that a combination of periostin overexpression and microvascular invasion in hepatocellular carcinoma was correlated with a poor prognosis and can be a good prognostic marker for hepatocellular carcinoma. (Gut Liver 2016;10:948-954)

Key Words: Periostin; Microvascular invasion; Hepatocellular carcinoma; Tissue microarray analysis; Prognosis

\section{INTRODUCTION}

Periostin, also known as osteoblast-specific factor 2, is an extracellular matrix protein that has been shown to be an important regulator of bone and tooth formation and maintenance. ${ }^{1}$ Periostin first identified in bone is a molecule secreted from the mouse osteoblastic cell line MC3T3-E1. ${ }^{2}$ The molecular structure of periostin is particularly highly homologous to $\beta$ ig-h3, which is known to promote cell adhesion and the spread of fibroblasts. ${ }^{3}$ Cancer progression is recognized not only by aberrant events occurring only in cancer cells but also by substances surrounding cancer cells secreted by various cells in the tumor microenvironment and signaling pathways induced by cancer and other cells. ${ }^{4}$

In recent human cancer studies, periostin has also been reported to be related to metastatic potential and poor prognosis in many kinds of cancer. Previous reports indicate that overexpression of periostin in tumor tissues can promote angiogenesis and invasion in various human cancers, including colon, head and neck, oral, nasopharyngeal, thyroid, breast, and pancreatic cancers. ${ }^{5-11}$ However, reports that support the association of periostin expression with the prognosis of hepatocellular carcinoma (HCC) and its clinical significance in HCC are lacking. ${ }^{12,13}$ We therefore aimed to analyze periostin expression in HCC and determine its implication in the prognosis of HCC.

\section{MATERIALS AND METHODS}

\section{Patients and tissue specimens}

We evaluated 149 HCC patients who underwent surgical resection as first-line treatment in Kyungpook National University

Correspondence to: Soo Young Park

Department of Internal Medicine, Kyungpook National University Hospital, 130 Dongdeok-ro, Jung-gu, Daegu 41944, Korea

Tel: +82-53-200-5505, Fax: +82-53-426-2046, E-mail: psyoung0419@gmail.com

Received on September 23, 2015. Revised on December 18, 2015. Accepted on February 5, 2016. Published online July 27, 2016 pISSN 1976-2283 eISSN 2005-1212 https://doi.org/10.5009/gnl15481

@ This is an Open Access article distributed under the terms of the Creative Commons Attribution Non-Commercial License (http://creativecommons.org/licenses/by-nc/4.0) which permits unrestricted non-commercial use, distribution, and reproduction in any medium, provided the original work is properly cited. 
Hospital between 2006 and 2010. HCC was diagnosed and treated according to the American Associated for the Study of Liver Diseases guidelines. ${ }^{14}$ We excluded patients who had received prior treatments such as local ablation therapy and transarterial chemoembolization. We also excluded HCC patients who had portal vein thrombosis and radiologic vascular invasion, which are not indicated for surgical resection. For more than one nodules distributed in one lobe, we could perform surgical resection.

Clinical data were obtained by reviewing the patients' medical records, including age, sex, tumor size, tumor number, laboratory results, and etiology of underlying liver disease. All of the patients were followed-up by using multiphasic dynamic computed tomography and serum $\alpha$-fetoprotein (AFP) measurement every 3 months. This study was approved by the Institutional Review Board of Kyungpook National University Hospital (KNUH-2014-04-056-001).

\section{Immunohistochemical staining}

All tissue specimens were formalin-fixed and paraffinembedded. We built sets of tissue microarrays from surgical specimens of HCC and nontumorous tissue for immunohistochemistry. Anti-Periostin antibody (Abcam, Cambridge, UK) was used for immunohistochemistry. Periostin expression was assessed according to staining intensity, and was scored as 1 to 3 as follows: (1) weakly positive; (2) moderately positive; and (3) strongly positive. For statistical analyses, the immunohistochemical staining scores were categorized as low (1 and 2) or high (3). Two specialized pathologists blinded to the patients' clinical data independently reviewed histopathological and immunochemistry results three times, for tumor histology, capsule invasion, microvascular invasion, and periostin expression. Microvascular invasion was defined as microscopic tumor invasion or tumor emboli within the central, portal, or hepatic vein or large capsular vessels.

\section{Statistical analysis}

Statistical analysis was performed using SPSS version 20.0 software for Windows (IBM Corp., Armonk, NY, USA). The chisquare or Fischer exact test was used to analyze the relationship between periostin expression level and clinicopathological char-
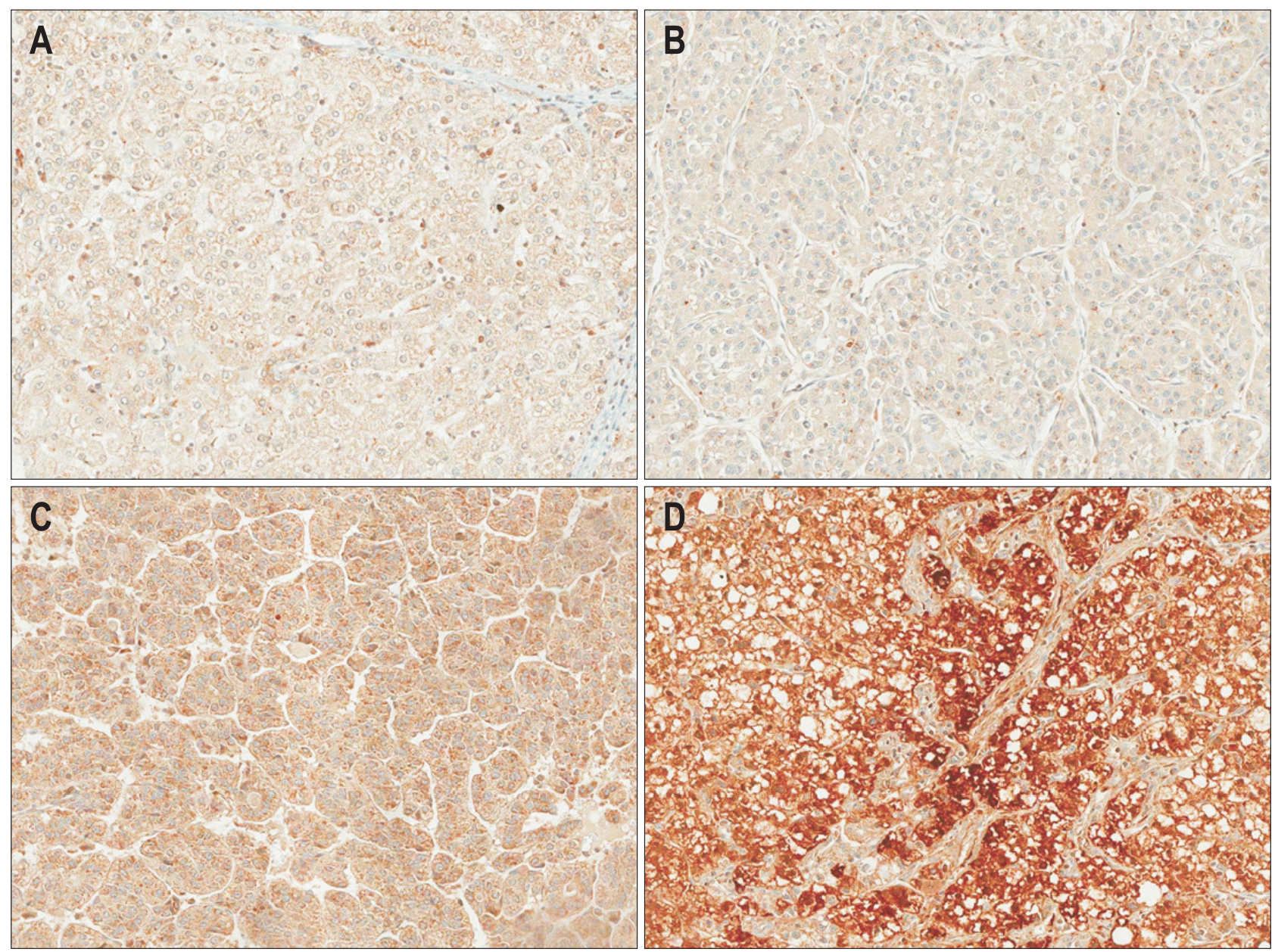

Fig. 1. Immunohistochemical staining of periostin (original magnification, $\times 200$ ) in normal $(A)$ and hepatocellular carcinoma tissues (B, weakly positive; C, moderately positive; $\mathrm{D}$, strongly positive). 
acteristics. Recurrence-free survival and overall survival were calculated from date of surgical resection to local or distant relapse, death, or last follow-up and plotted as Kaplan-Meier curves. The log-rank test was used for evaluating differences in overall and recurrence-free survivals. We included as variables periostin expression, microvascular invasion, combination of periostin expression and microvascular invasion, age, sex, modified Union for International Cancer Control (UICC) stage, tumor size, tumor number, presence of capsule, and AFP in the univariate analysis. In the multivariate analysis, we included the variables in the univariate analysis, but excluded the components of combination of periostin expression and microvascular invasion and modified UICC stage. The components were periostin, microvascular invasion, tumor number, and tumor size. The multivariate analyses were performed according to the Cox regression model.

\section{RESULTS}

\section{Expression pattern of periostin in clinical tissue specimens}

We analyzed periostin expression by immunohistochemistry in 149 surgically resected HCC tissues using tissue microarrays (Fig. 1). Periostin was predominantly expressed in HCC cells compared with corresponding normal hepatocytes, which was mainly observed in cytoplasmic area of HCC cells.

\section{Periostin expression in HCC tissues and its correlations with clinicopathological characteristics}

Baseline characteristics of the patients are summarized in Table 1. There were 111 male patients (74.5\%) and 38 female patients $(25.5 \%)$, with a median age of 56 years (range, 14 to 78 years). The median tumor size was $3.5 \mathrm{~cm}$ (range, 1.2 to 14.5 $\mathrm{cm})$. The causes of underlying liver disease were hepatitis $B$ virus infection ( $\mathrm{n}=115,77.2 \%)$, alcohol consumption $(\mathrm{n}=14$, $12.1 \%)$, hepatitis C virus infection ( $\mathrm{n}=13,8.7 \%)$, and cryptogenic $(\mathrm{n}=7,2.0 \%)$. The mean follow-up period was 48.5 months (range, 6 to 60 months).

Sixty-two patients (41.6\%) showed high periostin expression levels in HCC tissues, whereas 87 patients (58.4\%) showed low periostin expression levels in HCC tissues. To determine the association between the periostin expression level and clinical characteristics, differences in baseline clinicopathological characteristics according to the periostin expression level are described in Table 1. A high periostin level was observed more frequently in case of multiple tumors (odds ratio [OR], 2.826; 95\% confidence interval [CI], 1.224 to 6.527 ; $\mathrm{p}=0.013$ ), positive microvascular invasion (OR, 2.974; 95\% CI, 1.431 to 6.181 ; $\mathrm{p}=0.003)$, and an advanced modified UICC stage (OR, 3.032; 95\% CI, 1.424 to $6.452 ; \mathrm{p}=0.003)$. Age, sex, etiology of underlying liver disease, Child-Pugh class, tumor size, Edmondson grade, AFP level, and existence of a tumor capsule were not correlated with the periostin expression level.

\section{High periostin expression associates with poor survival of HCC patients}

The cumulative 1-, 3-, and 5-year overall survival rates were 97.7\%, 85.1\%, and 77.5\%, respectively, and the corresponding recurrence-free survival rates were 92.0\%, 65.1\%, and 50.9\%,

Table 1. Periostin Expression Levels and Clinicopathologic Features of 149 Hepatocellular Carcinoma Cases

\begin{tabular}{|c|c|c|c|c|}
\hline \multirow{2}{*}{ Clinicopathologic data } & \multirow{2}{*}{$\begin{array}{l}\text { No. of } \\
\text { cases }\end{array}$} & \multicolumn{2}{|c|}{ Periostin expression } & \multirow{2}{*}{ p-value } \\
\hline & & Low & High & \\
\hline Age, yr & & & & 0.945 \\
\hline$\leq 56$ & 75 & 44 & 31 & \\
\hline$>56$ & 74 & 43 & 31 & \\
\hline Sex & & & & 0.146 \\
\hline Male & 111 & 61 & 50 & \\
\hline Female & 38 & 26 & 12 & \\
\hline Etiology & & & & 0.690 \\
\hline $\mathrm{HBV}$ & 115 & 69 & 46 & \\
\hline $\mathrm{HCV}$ & 13 & 6 & 7 & \\
\hline Alcohol & 18 & 10 & 8 & \\
\hline Cryptogenic & 3 & 2 & 1 & \\
\hline Child-Pugh class & & & & $1.000^{*}$ \\
\hline A & 147 & 61 & 86 & \\
\hline $\mathrm{B}$ & 2 & 1 & 1 & \\
\hline Tumor size, $\mathrm{cm}$ & & & & 0.236 \\
\hline$\leq 5$ & 104 & 64 & 40 & \\
\hline$>5$ & 45 & 23 & 22 & \\
\hline Tumor no. & & & & $0.013^{\dagger}$ \\
\hline Single & 120 & 76 & 44 & \\
\hline Multiple & 29 & 11 & 18 & \\
\hline Microvascular invasion & & & & $0.003^{\dagger}$ \\
\hline Yes & 43 & 17 & 26 & \\
\hline No & 106 & 70 & 36 & \\
\hline Edmondson grade & & & & 0.560 \\
\hline I-II & 93 & 56 & 37 & \\
\hline III-IV & 56 & 31 & 25 & \\
\hline Serum $\alpha$-fetoprotein, $\mathrm{ng} / \mathrm{mL}$ & & & & 0.578 \\
\hline$\leq 200$ & 102 & 58 & 44 & \\
\hline$>200$ & 47 & 29 & 18 & \\
\hline Modified UICC stage & & & & $0.003^{\dagger}$ \\
\hline I-II & 110 & 72 & 38 & \\
\hline III-IV & 39 & 15 & 24 & \\
\hline Tumor capsule & & & & 0.806 \\
\hline Yes & 92 & 53 & 39 & \\
\hline No & 57 & 34 & 23 & \\
\hline
\end{tabular}

HBV, hepatitis B virus; HCV, hepatitis C virus; UICC, Union for International Cancer Control.

${ }^{*}$ Fischer exact test; ${ }^{\dagger} \mathrm{p}<0.05$. 
A

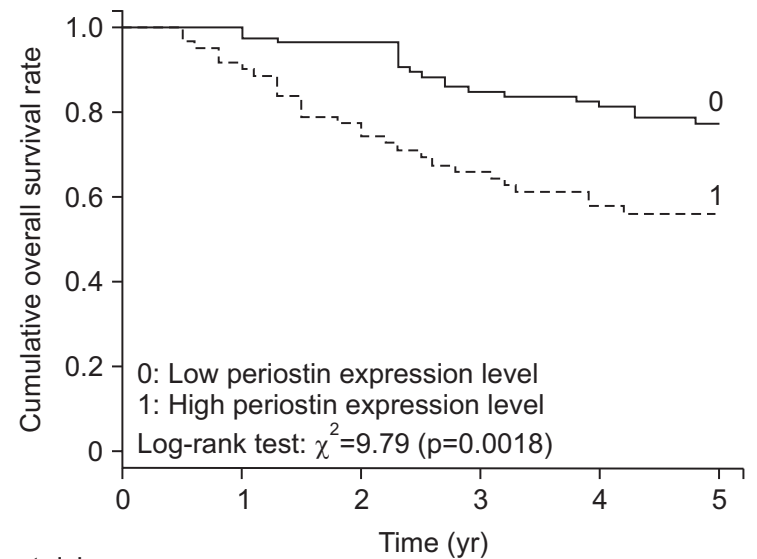

No. at risk

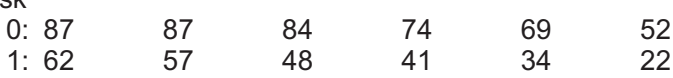

B

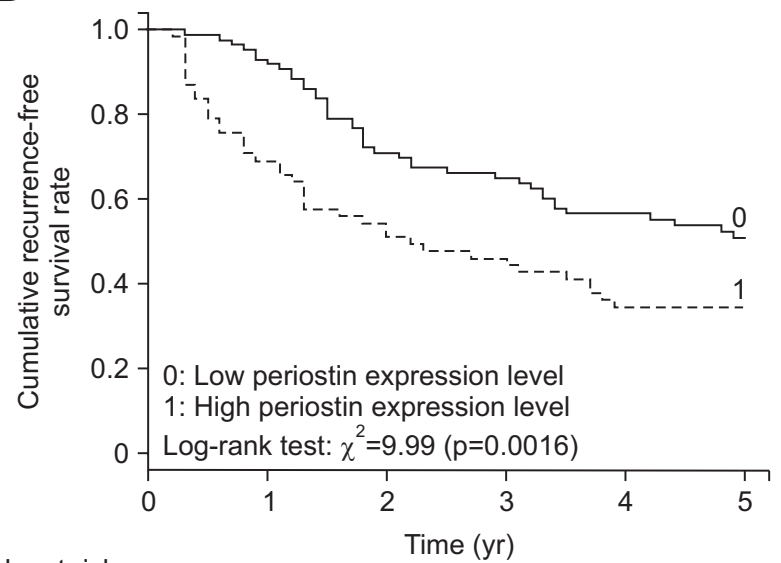

No. at risk

$\begin{array}{llllll}0: 87 & 81 & 61 & 54 & 45 & 33 \\ \text { 1: } 62 & 42 & 33 & 28 & 20 & 11\end{array}$

Fig. 2. Kaplan-Meier analysis of overall survival (A) and recurrence-free survival (B) curves of patients with hepatocellular carcinoma based on periostin expression levels.

respectively. Patients with a high periostin expression level had significantly $(\mathrm{p}=0.002)$ lower overall survival rates $(90.3 \%$, $66.1 \%$, and 56.2\% at 1-, 3-, and 5-year) than did those with a low periostin expression level $(97.7 \%, 85.1 \%$, and $77.5 \%$ at 1-, $3-$, and 5-year). Similarly, patients with a high periostin expression level had significantly $(\mathrm{p}=0.006)$ lower overall survival rates (83.9\%, 42.8\%, and 34.6\% at 1-, 3-, and 5-year) than did those with a low periostin expression level (92.0\%, 62.7\%, and 50.9\% at 1-, 3-, and 5-years) (Fig. 2).

\section{Combination of periostin expression and microvascular invasion is an independent prognostic factor for survival in HCC patients}

The periostin expression level and microvascular invasion were significantly and negatively correlated with overall survival and recurrence-free survival, respectively. Therefore, we developed another variable, periostin expression level×microvascular invasion, with a low periostin expression level and negative microvascular invasion scored as 0 , a high periostin expression level and negative microvascular invasion scored as 1; a low periostin expression level and positive microvascular invasion scored as 2 ; and a high periostin expression level and positive microvascular invasion scored as 3 .

Tables 2 and 3 display the results of the univariate and multivariate analyses for identifying prognostic factors that affect the overall survival and recurrence-free survival of the 149 HCC patients. In a univariate analysis, the high periostin expression level ( $p=0.021$ ), positive microvascular invasion $(p=0.001)$, high periostin expression level $\times$ positive microvascular invasion ( 1 vs $0, p=0.001 ; 2$ vs $0, p<0.001 ; 3$ vs $0, p<0.001$ ), male $\operatorname{sex}(p=0.014)$, large tumor size ( $>5 \mathrm{~cm}, \mathrm{p}=0.018$ ), and an advanced modified UICC stage (III vs I, $\mathrm{p}=0.011$ ) were significant prognostic factors for the overall survival of the HCC patients (Table 2). A high periostin expression level ( $\mathrm{p}=0.026)$, positive microvascular invasion $(\mathrm{p}=0.005)$, high periostin expression level $\times$ positive microvascular invasion ( 3 vs $0, p=0.001)$, male sex $(p=0.043$ ), multiple tumor number ( $\mathrm{p}=0.013$ ), and an advanced modified UICC stage (III vs I, $\mathrm{p}=0.011$ ) were significant prognostic factors of the recurrence-free survival of the HCC patients (Table 3).

In a multivariate analysis, only high periostin expression level×positive microvascular invasion ( 1 vs $0, p=0.002 ; 2$ vs 0 , $\mathrm{p}=0.001$; 3 vs $0, \mathrm{p}=0.006$ ) was relevant to overall survival (Table 2). In addition, only high periostin expression level×positive microvascular invasion ( 3 vs $0, p=0.025$ ) was relevant to recurrence-free survival (Table 3). The 1-, 3-, and 5-year cumulative overall survival rates were 96.7\%, 90.0\%, and 79.7\% for the low periostin expression level $\times$ negative microvascular invasion patients, $89.5 \%, 57.9 \%$, and $42.1 \%$ for the high periostin expressionxpositive microvascular invasion patients. The 1-, 3-, and 5-year cumulative recurrence-free survival rates were $90.0 \%, 30.0 \%$, and $0.0 \%$ for the low periostin expression level×negative microvascular invasion patients, 47.4\%, 10.5\%, and $0.0 \%$ for the high periostin expression $\times$ positive microvascular invasion patients. High periostin expression level×positive microvascular invasion patient group showed significantly lower overall survival rates $(\mathrm{p}<0.001)$ and recurrence-free survival rates $(p=0.010)$ than low periostin expression $\times$ positive microvascular invasion patient group showed (Fig. 3).

\section{DISCUSSION}

A high periostin expression level is known to be associated with a poor prognosis of many cancers. ${ }^{15}$ Their molecular mechanism is not fully understood, but recent studies revealed that periostin binding to integrins activates the focal adhesion kinase (FAK)- and Akt (also known as protein kinase B, PKB)-mediated 
Table 2. Univariate and Multivariate Analyses of Factors Associated with Overall Survival

\begin{tabular}{|c|c|c|c|c|}
\hline & \multicolumn{2}{|c|}{ Univariate analysis } & \multicolumn{2}{|c|}{ Multivariate analysis } \\
\hline & HR (95\% CI) & $\mathrm{p}$-value & HR $(95 \%$ CI) & p-value \\
\hline Periostin expression level (high vs low) & $1.982(1.111-3.536)$ & $0.021^{*}$ & & \\
\hline MVI (yes vs no) & $2.657(1.488-4.741)$ & $0.001^{*}$ & & \\
\hline \multicolumn{5}{|l|}{$\mathrm{PO} \times \mathrm{MVI}^{\dagger}$} \\
\hline \multicolumn{5}{|l|}{0} \\
\hline 1 & $4.126(1.804-9.437)$ & $0.001^{*}$ & $3.754(1.621-8.693)$ & $0.002^{*}$ \\
\hline 2 & $5.597(2.271-13.792)$ & $<0.001^{*}$ & $4.833(1.928-12.119)$ & $0.001^{*}$ \\
\hline 3 & $4.681(1.971-11.118)$ & $<0.001^{*}$ & $3.452(1.424-8.366)$ & $0.006^{*}$ \\
\hline Age, yr (>56 vs $\leq 56$ ) & $0.963(0.529-1.753)$ & 0.903 & & \\
\hline Sex (female vs male) & $0.313(0.124-0.793)$ & $0.014^{*}$ & $0.390(0.149-1.020)$ & 0.055 \\
\hline Tumor size, $\mathrm{cm}$ (>5 vs $\leq 5$ ) & $2.025(1.130-3.629)$ & $0.018^{*}$ & & \\
\hline Tumor no. (multiple vs single) & $1.572(0.814-3.037)$ & 0.178 & & \\
\hline \multicolumn{5}{|l|}{ Tumor stage (modified UICC) } \\
\hline \multicolumn{5}{|l|}{$\mathrm{I}$} \\
\hline II & $5.818(0.788-42.94)$ & 0.084 & $5.939(0.791-44.563)$ & 0.083 \\
\hline III & $13.44(1.802-100.18)$ & $0.011^{*}$ & $7.679(1.003-58.815)$ & 0.050 \\
\hline Presence of capsule (yes vs no) & $1.081(0.594-1.967)$ & 0.800 & & \\
\hline AFP, ng/mL (>200 vs $\leq 200)$ & $0.750(0.388-1.449)$ & 0.392 & & \\
\hline
\end{tabular}

HR, hazard ratio; CI, confidence interval; MVI, microvascular invasion; PO, periostin; UICC, Union for International Cancer Control; AFP, $\alpha$-fetoprotein.

*p<0.05; ${ }^{\dagger} 0$ : low P0×negative MVI; 1: high PO×negative MVI; 2: low PO×positive MVI; 3: high PO×positive MVI.

Table 3. Univariate and Multivariate Analyses of Factors Associated with Recurrence-Free Survival

\begin{tabular}{|c|c|c|c|c|}
\hline & \multicolumn{2}{|c|}{ Univariate analysis } & \multicolumn{2}{|c|}{ Multivariate analysis } \\
\hline & $\mathrm{HR}(95 \% \mathrm{CI})$ & p-value & HR $(95 \%$ CI) & p-value \\
\hline Periostin expression level (high vs low) & $1.645(1.062-2.548)$ & $0.026^{*}$ & & \\
\hline MVI (yes vs no) & $1.908(1.214-3.000)$ & $0.005^{*}$ & & \\
\hline \multicolumn{5}{|l|}{$\mathrm{PO} \times \mathrm{MVI}^{\dagger}$} \\
\hline \multicolumn{5}{|l|}{0} \\
\hline 1 & $1.713(0.980-2.993)$ & 0.059 & $1.491(0.844-2.635)$ & 0.169 \\
\hline 2 & $1.816(0.909-3.627)$ & 0.091 & $1.730(0.846-3.537)$ & 0.133 \\
\hline 3 & $2.731(1.534-4.861)$ & $0.001^{*}$ & $2.029(1.095-3.759)$ & $0.025^{*}$ \\
\hline Age, yr (>56 vs $\leq 56$ ) & $0.952(0.606-1.495)$ & 0.831 & & \\
\hline Sex (female vs male) & $0.561(0.320-0.983)$ & $0.043^{*}$ & $0.635(0.354-1.137)$ & 0.127 \\
\hline Tumor size, $\mathrm{cm}$ ( $>5$ vs $\leq 5$ ) & $1.362(0.857-2.162)$ & 0.191 & & \\
\hline Tumor no. (multiple vs single) & $1.874(1.139-3.083)$ & $0.013^{*}$ & & \\
\hline \multicolumn{5}{|l|}{ Tumor stage (modified UICC) } \\
\hline \multicolumn{5}{|l|}{ I } \\
\hline II & $1.007(0.490-2.070)$ & 0.984 & $0.851(0.405-1.791)$ & 0.671 \\
\hline III & $2.623(1.243-5.535)$ & $0.011^{*}$ & $1.775(0.794-3.971)$ & 0.162 \\
\hline Presence of capsule (yes vs no) & $0.952(0.609-1.486)$ & 0.828 & & \\
\hline AFP, ng/mL (>200 vs $\leq 200$ ) & $0.931(0.581-1.491)$ & 0.765 & & \\
\hline
\end{tabular}

HR, hazard ratio; CI, confidence interval; MVI, microvascular invasion; PO, periostin; UICC, Union for International Cancer Control; AFP, $\alpha$-fetoprotein.

${ }^{*} \mathrm{p}<0.05$; ${ }^{\dagger} 0$ : low POxnegative MVI; 1: high P0×negative MVI; 2: low POxpositive MVI; 3: high P0xpositive MVI. 
A

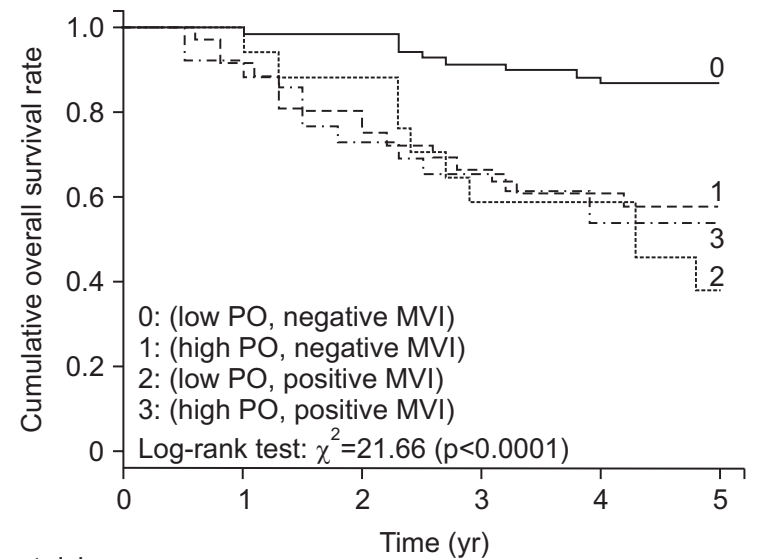

No. at risk

$\begin{array}{lllllr}\text { 0: } 70 & 70 & 69 & 64 & 59 & 47 \\ \text { 1: } 36 & 33 & 29 & 24 & 21 & 12 \\ \text { 2: } 17 & 17 & 15 & 10 & 10 & 5 \\ \text { 3: } 26 & 24 & 19 & 17 & 13 & 10\end{array}$

B

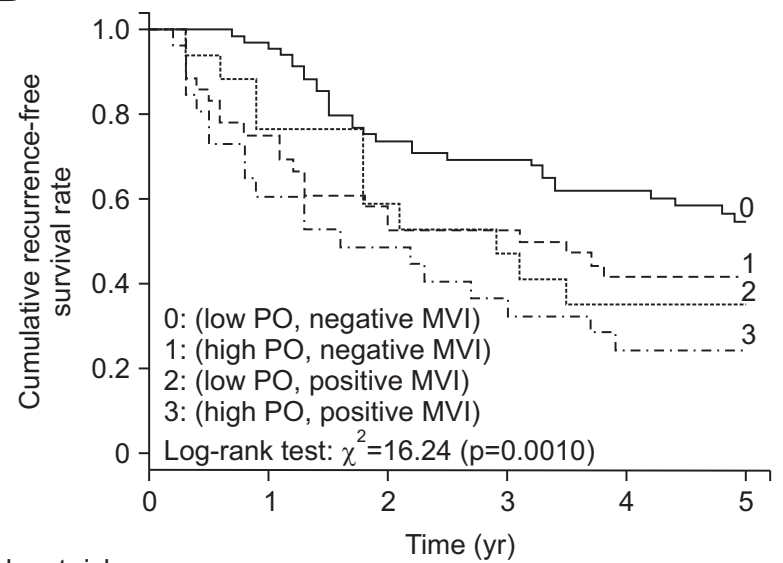

No. at risk

$\begin{array}{rrrrrr}\text { 0: } 70 & 68 & 51 & 46 & 39 & 28 \\ \text { 1: } 36 & 27 & 21 & 19 & 15 & 9 \\ \text { 2: } 17 & 13 & 10 & 8 & 6 & 5 \\ \text { 3: } 26 & 15 & 12 & 9 & 5 & 2\end{array}$

Fig. 3. Kaplan-Meier analysis of overall survival (A) and recurrence-free survival (B) curves of patients with hepatocellular carcinoma based on the combination of periostin expression levels and microvascular invasion.

PO, periostin; MVI, microvascular invasion.

signaling pathways which promote tumor angiogenesis, invasion and metastasis. ${ }^{4,16}$ According to the laboratory finding from one study, ${ }^{10}$ periostin promotes tumor angiogenesis and metastasis through upregulation of vascular endothelial growth factor (VEGF) receptor 2 expression. In addition, HCC in the periostinpositive group showed higher VEGF expression levels and higher microvascular density than HCC in the periostin-negative group, ${ }^{12}$ which is supported by the fact that VEGF stimulates endothelial cell proliferation and induces the formation of new blood vessels in HCC. ${ }^{17}$

We think that the correlation of the periostin expression level with microvascular invasion is probably the consequence of epithelial-mesenchymal transition. Periostin is a mesenchymal marker of cancer cells with other marker proteins such as vimentin, fibronectin, and N-cadherin. Similarly, a previous study showed that liver-intestine cadherin expression predicts microvascular invasion and a poor prognosis in hepatitis B viruspositive HCC. ${ }^{18}$ Periostin probably exerts its pro-tumorigenic effect through its binding to the integrins and the consequent activation of intracellular pathways, which determine enhanced invasiveness. ${ }^{19}$ This should be further studied with HCC cell lines.

In this study, we found that the periostin expression level was correlated with overall survival and recurrence-free survival in HCC. An especially high periostin expression level was correlated with microvascular invasion. Microvascular invasion has been regarded in many studies as an independent predictor of recurrence and poorer survival after hepatic resection for HCC. ${ }^{20-23}$ In our study, we found that even if microvascular invasion did not exist, the periostin expression level could predict prognosis. In addition, a combination of the two factors could predict overall and recurrence-free survival better than the two factors considered alone.

We reviewed a total of 149 HCC cases, a relatively large number of cases compared with that in previously published studies. However, this study has several limitations. First, this was a retrospective study, so clinical data were based on medical records. Patients with missing medical records were excluded from the analysis. Second, this was a single-center study, and hence, the results might not be generalizable to a broader population. In addition, the etiology of liver disease being predominantly hepatitis B virus infection limits the generalization of the results. Third, tissue periostin can be used as a biomarker for prognosis prediction, but tissue samples were obtained through percutaneous liver biopsy or liver resection, which is invasive and increases the risk to patients. Other noninvasive diagnostic approaches for the determination of periostin expression are needed to verify its validity as a prognostic marker. Fourth, our study was performed to investigate the relationship between periostin expression and patient prognosis, and not to reveal the mechanism of angiogenesis by periostin. Further studies on this issue are needed.

In conclusion, a high periostin expression level in HCC tissues suggested a poor prognosis and was correlated with microvascular invasion, multiple tumors, and an advanced modified UICC stage. Especially, the combination of periostin expression level and microvascular invasion is a better predictor of prognosis of HCC patients.

\section{CONFLICTS OF INTEREST}

No potential conflict of interest relevant to this article was 
reported.

\section{ACKNOWLEDGEMENTS}

This study was supported by Biomedical Research Institute grant, Kyungpook National University Hospital, 2014 (number: H00082-14-13).

\section{REFERENCES}

1. Horiuchi K, Amizuka N, Takeshita S, et al. Identification and characterization of a novel protein, periostin, with restricted expression to periosteum and periodontal ligament and increased expression by transforming growth factor beta. J Bone Miner Res 1999;14:1239-1249.

2. Takeshita S, Kikuno R, Tezuka K, Amann E. Osteoblast-specific factor 2: cloning of a putative bone adhesion protein with homology with the insect protein fasciclin I. Biochem J 1993;294(Pt 1):271-278.

3. Litvin J, Selim AH, Montgomery MO, et al. Expression and function of periostin-isoforms in bone. J Cell Biochem 2004;92:10441061.

4. Utispan K, Sonongbua J, Thuwajit P, et al. Periostin activates integrin $\alpha 5 \beta 1$ through a PI3K/AKT-dependent pathway in invasion of cholangiocarcinoma. Int J Oncol 2012;41:1110-1118.

5. Bao S, Ouyang G, Bai X, et al. Periostin potently promotes metastatic growth of colon cancer by augmenting cell survival via the Akt/PKB pathway. Cancer Cell 2004;5:329-339.

6. Kudo Y, Ogawa I, Kitajima S, et al. Periostin promotes invasion and anchorage-independent growth in the metastatic process of head and neck cancer. Cancer Res 2006;66:6928-6935.

7. Siriwardena BS, Kudo Y, Ogawa I, et al. Periostin is frequently overexpressed and enhances invasion and angiogenesis in oral cancer. Br J Cancer 2006;95:1396-1403.

8. Chang Y, Lee TC, Li JC, et al. Differential expression of osteoblastspecific factor 2 and polymeric immunoglobulin receptor genes in nasopharyngeal carcinoma. Head Neck 2005;27:873-882.

9. Fluge Ø, Bruland 0, Akslen LA, Lillehaug JR, Varhaug JE. Gene expression in poorly differentiated papillary thyroid carcinomas. Thyroid 2006;16:161-175.

10. Shao R, Bao S, Bai X, et al. Acquired expression of periostin by human breast cancers promotes tumor angiogenesis through upregulation of vascular endothelial growth factor receptor 2 expression. Mol Cell Biol 2004;24:3992-4003.

11. Baril P, Gangeswaran R, Mahon PC, et al. Periostin promotes in- vasiveness and resistance of pancreatic cancer cells to hypoxia-induced cell death: role of the beta4 integrin and the PI3k pathway. Oncogene 2007;26:2082-2094.

12. Lv Y, Wang W, Jia WD, et al. High-level expression of periostin is closely related to metastatic potential and poor prognosis of hepatocellular carcinoma. Med Oncol 2013;30:385.

13. Lv Y, Wang W, Jia WD, et al. High preoparative levels of serum periostin are associated with poor prognosis in patients with hepatocellular carcinoma after hepatectomy. Eur J Surg Oncol 2013;39:1129-1135.

14. Bruix J, Sherman M; Practice Guidelines Committee, American Association for the Study of Liver Diseases. Management of hepatocellular carcinoma. Hepatology 2005;42:1208-1236.

15. Wang W, Sun QK, He YF, et al. Overexpression of periostin is significantly correlated to the tumor angiogenesis and poor prognosis in patients with esophageal squamous cell carcinoma. Int J Clin Exp Pathol 2014;7:593-601.

16. Li G, Jin R, Norris RA, et al. Periostin mediates vascular smooth muscle cell migration through the integrins alphavbeta3 and alphavbeta5 and focal adhesion kinase (FAK) pathway. Atherosclerosis 2010;208:358-365.

17. Suzuki K, Hayashi N, Miyamoto Y, et al. Expression of vascular permeability factor/vascular endothelial growth factor in human hepatocellular carcinoma. Cancer Res 1996;56:3004-3009.

18. Ding ZB, Shi YH, Zhou J, et al. Liver-intestine cadherin predicts microvascular invasion and poor prognosis of hepatitis B viruspositive hepatocellular carcinoma. Cancer 2009;115:4753-4765.

19. Morra L, Moch H. Periostin expression and epithelial-mesenchymal transition in cancer: a review and an update. Virchows Arch 2011;459:465-475.

20. Wang CC, Iyer SG, Low JK, et al. Perioperative factors affecting long-term outcomes of 473 consecutive patients undergoing hepatectomy for hepatocellular carcinoma. Ann Surg Oncol 2009;16:1832-1842.

21. Sumie S, Kuromatsu R, Okuda K, et al. Microvascular invasion in patients with hepatocellular carcinoma and its predictable clinicopathological factors. Ann Surg Oncol 2008;15:1375-1382.

22. Fan ST, Poon RT, Yeung C, et al. Outcome after partial hepatectomy for hepatocellular cancer within the Milan criteria. Br J Surg 2011;98:1292-1300.

23. Lim KC, Chow PK, Allen JC, et al. Microvascular invasion is a better predictor of tumor recurrence and overall survival following surgical resection for hepatocellular carcinoma compared to the Milan criteria. Ann Surg 2011;254:108-113. 